\title{
Amino Acids in Boron Neutron Capture Therapy-Prospects for Precise Treatment of Malignant Brain Tumors
}

\author{
Juri M. Timonen* \\ School of Pharmacy, Faculty of Health Sciences, University of Eastern Finland, P. O. Box 1627, \\ 70211 Kuopio, Finland \\ Email: juri.timonen@uef.fi (J. M. T.)
}

\begin{abstract}
During the last 60 years, a great number of boron-containing compounds have been synthesized and designated as potential boron carriers for boron neutron capture therapy (BNCT) of several types of cancer. Several of these putative boron carriers are derivatives of $\alpha$-amino acids (AAs). Despite sixty years of research, there are only two compounds used in the clinic including boronophenylalanine (BPA). Cancer cells have long been known to consume enormous amounts of nutrients such as AAs. Recent studies have revealed many mechanisms, through which they fulfill their demand for nutrients. The large amino acid transporter 1 (LAT1) is an extensively studied transporter. Recent studies have expanded our knowledge and understanding of the structure of LAT1 and its mode of action. It has been demonstrated that BPA and some other AA-based boron carriers are transported by LAT1 into cancer cells. In summary, these advances have opened new prospects for designing novel LAT1-targeted AA-based boron carriers for malignant brain tumors.
\end{abstract}

Keywords boron neutron capture therapy, cancer, amino acid, LAT1, glioma

\section{Introduction}

Cancer is one of the most devastating diseases. In 2018, it was estimated that around 270,000 individuals had died from cancer of the brain and nervous system. ${ }^{[1]}$ It would be advantageous to develop a very selectively targeted cancer therapy that could destroy a single malignant cell while sparing the adjacent healthy cell. This would change the course of cancer treatment. Over 60 years ago, such a therapy was invented but only recently has a technical development made it possible to exploit this technique in hospitals. This method is called boron neutron capture therapy (BNCT).

In $\mathrm{BNCT}$, the patient is given a boron carrier $\left({ }^{10} \mathrm{~B}\right.$ containing tumor-targeted compound). After enough boron has accumulated into the tumor, the patient is exposed to neutron irradiation. Thermal neutrons will interact only with the nuclei of ${ }^{10} \mathrm{~B}$ atoms that can capture a neutron and undergo nuclear fission. In this fission process, an energy rich a-particle and a lithium ion are produced inside the cell. The collision of these particles with crucial molecules, e.g., DNA, will damage the cell and lead to cell death. ${ }^{[2]}$

For decades, the challenge of hospital use of BNCT has been the lack of an efficient and safe neutron source. Recent progress in the development of a linear accelerator-based neutron source has led to the installation of the world's first hospital-based BNCT device in the University Hospital of Helsinki, Finland. The next step towards effective BNCT for the treatment of malignant brain tumors has been the development of more selective boron carriers. ${ }^{[3]}$

\section{Amino Acid Based BNCT}

Amino acids (AAs) are essential nutrients for all growing cells, especially for cancer cells. They are transported into cells by different amino acid transporters, from which one particular transporter, namely large amino acid transporter 1 (LAT1), has shown good potential as a target for efficient and targeted drug delivery to brain tumors. LAT1 is highly expressed in the blood-brain barrier (BBB) that protects the brain from detrimental compounds but allows the passage of nutrients. Importantly, the expression of LAT1 is also significantly upregulated in some human cancer types. ${ }^{[4]}$

During the past 60 years, several AA-based boron carriers have been synthesized, but only a few have been biologically evaluated. One of the best studied compounds, boronophenylalanine (BPA), has been confirmed to be transported via LAT1. ${ }^{[5,6]}$ The extensive research examining how AAs can be exploited in a prodrug approach, has revealed details of how AAs are transported into cancer cells. These results can be utilized directly in the design and development of novel boron carriers. ${ }^{[7]}$

\section{Structure and Synthesis of Boronated Amino Acids}

The synthesis of BPA was firstly published in $1958 .{ }^{[8]}$ Subsequently, a vast number of different borons containing AAs have been described. When classified by the nature of $A A$, they can be divided into two classes: aromatic amino acids like BPA (1) ${ }^{[7,8]}$ and aliphatic amino acids like 2-amino-4-boronobutyric acid (2). ${ }^{[9]}$ Boron can be attached to the amino acid as a boronic acid (1 and $\mathbf{2}$ ) or as a boron cluster, e.g., carboranyl (3) (Figure 1). ${ }^{[10]}$<smiles>NC(Cc1ccc(B(O)O)cc1)C(=O)O</smiles>

$\mathrm{OH} \quad(1)$<smiles>NC(CCB(O)O)C(=O)O</smiles>

(2)<smiles>N[C@@H](C[C@@H]1[CH]C1)C(=O)O</smiles>

(3)

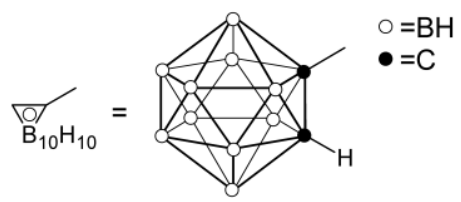

Figure 1 Structures of BPA and some other boron carriers for BNCT. 


\section{Perspective}

Several approaches have been utilized to prepare boronated AAs (Figure 2). Nowadays, the main route to make AAs bearing a boron-group attached to aromatic ring is to exploit Miasaki-cross coupling. However, it is not possible to use the straight substitution of phenolic $\mathrm{OH}$ via triflation and substitution of the triflate to borono group in the synthesis of 5 -boronated tryptophan. Thus, this synthesis has been achieved by the Friedels-Crafts alkylation of 5-borono indole. ${ }^{[11]}$

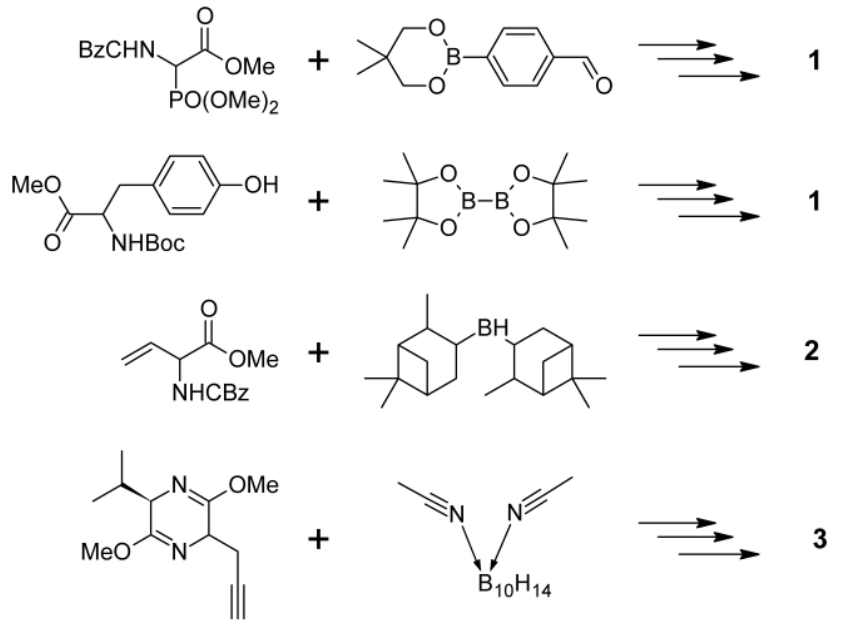

Figure 2 Different synthetic route leading to boronated AAs.

\section{Challenges of Boron}

Many of the synthetized AA based boron carriers are boronic acid derivatives and their solubility has proved challenging. In clinical trials, conjugates of BPA with fructose have been used to overcome the solubility problems. ${ }^{[12]}$

The analyses of uptake, localization and accumulation of boron inside cells are complicated. However, the methods using inductively coupled plasma mass spectrometry (ICP-MS) and NMR have been developed, which can analyze the amount of boron in biological samples. ${ }^{[13,14]}$ The location of boron inside cells also plays an important role. The closer the fission occurs within the cell's nuclei, the more it damages the DNA. It increases the probability that the cell will die. Studies have also been conducted with secondary ion mass spectrometry (SIMS) to estimate the distribution of boron inside the cell. ${ }^{[15]}$

\section{Prospects of LAT1-Targeted Boron Carriers}

In recent decades, ${ }^{[16]}$ Finland has become a European pioneer in BNCT research and the installation of the world's first hospital-based BNCT device to Helsinki will initiate a new era of cancer treatment. ${ }^{[17]}$ The next decades will certainly see similar devices appearing in other hospitals, and many more treatments will be delivered for patients suffering from brain tumors and other malignant tumors.

Recent research on LAT1 ${ }^{[18]}$ has extended the knowledge of LAT1's 3D-structure and mode of action. It is predicted that ongoing studies will lead to the development of reliable computational models for designing and screening potential LAT1 substrates. As boronated AAs share a high structural similarity with natural AAs, the accumulating knowledge of LAT1's mode of action will form a basis for developing an even more specific design of boron carriers in the near future. Clearly, the biological evaluation of synthetic boron carriers will shape the landscape of LAT1 targeted BNCT in the next five to ten years.

\section{Conclusions}

Rapid progress in research of LAT1 has revealed the huge potential of using AAs in cancer targeting. Breakthroughs have occurred with the recent developments in hospital-based BNCT-devices and new analytical methods for studying the uptake, distribution and localization of boron in cells both in vitro and in vivo. It is likely that AA-based boron carriers will represent the next breakthrough in the treatment of brain tumor.

\section{Acknowledgements}

The Finnish Cultural Foundation and Academy of Finland (Project 308329) are acknowledged for funding BNCT-research. The School of Pharmacy, University of Eastern Finland is acknowledged for the facilities and the opportunity to conduct the research. Dr. Ewen MacDonald is thanked for improving this article's linguistic style. Professor Jarkko Rautio is acknowledged for his valuable comments about the article.

\section{References}

[1] Ferlay, J.; Colombet, M.; Soerjomataram, I.; Mathers, C.; Parkin, D. M.; Piñeros, M.; Znaor, A.; Bray, F. Int. J. Cancer 2019, 144, 1941.

[2] Miyatake, S. I.; Kawabata, S.; Hiramatsu, R.; Kuroiwa, T.; Suzuki, M.; Kondo, N.; Ono, K. Neurol. Med. Chir. (Tokyo) 2016, 56, 361.

[3] Yu, T. Ther. Radiol Oncol. 2018, 2, 63.

[4] Ylikangas, H.; Malmioja, K.; Peura, L.; Gynther, M.; Nwachukwu, E. O.; Leppänen, J.; Laine, K.; Rautio, J.; Lahtela-Kakkonen, M.; Huttunen, M. K.; Poso, A. ChemMedChem 2014, 9, 2699.

[5] Detta, A.; Cruickshank, G. S. Cancer Res. 2009, 69, 2126

[6] Wongthai, P.; Hagiwara, K.; Miyoshi, Y.; Wiriyasermkul, P.; Wei, L.; Ohgaki, R.; Kato, I.; Hamase, K.; Nagamori, S.; Kanai. Y. Cancer Sci. 2015, 106, 279.

[7] Rautio, J.; Meanwell, N. A.; Di, L.; Hageman, M. J. Nat. Rev. Drug Disc. 2018, 17, 559.

[8] Snyder, H. R., Albert J. Reedy, Wm J. Lennarz. J. Am. Chem. Soc. 1958, 80, 835.

[9] Denniel, V.; Bauchat, P.; Danion, D.; Danion-Bougot, R. Tetrahedron Lett. 1996, 37, 5111.

[10] Karnbrock, W.; Musiol, H. J.; Moroder, L. Tetrahedron 1995, 51, 1187.

[11] Bartolucci, S.; Bartoccini, F.; Righi, M.; Piersanti, G. Org. Lett. 2011, 14, 600.

[12] Bendel, P.; Anderson, C.; Kabalka, G. W. In Frontiers in Neutron Capture Therapy. Eds.: Hawthorne, M. F.; Shelly, K.; Wiersema, R. J., Springer, Boston, MA, 2001, pp. 869-874.

[13] Linko, S.; Revitzer, H.; Zilliacus, R.; Kortesniemi, M.; Kouri, M.; Savolainen, S. Scand. J. Clin. Lab. Invest. 2008, 68, 696.

[14] Bendel, P. NMR Biomed. 2005, 18, 74.

[15] Chandra, S.; Ahmad, T.; Barth, R. F.; Kabalka, G. W. J. Microsc. 2014, 254, 146.

[16] Savolainen, S.; Kortesniemi, M.; Timonen, M.; Reijonen, V.; Kuusela, L.; Uusi-Simola, J.; Välimäki, P. Phys. Medica 2013, 29, 233.

[17] Web page of the HUS-BNCT Project, accessed 31.7.2019. https://www.hus.fi/en/about-hus/Hospital_areas/Comprehensive-C ancer-Center/Pages/BNCT-Project.aspx

[18] Yan, R.; Zhao, X.; Lei, J.; Zhou, Q. Nature 2019, 568, 127.

Received August 16, 2019 Accepted August 30, 2019 